\title{
Experimental System for Real-Time Motion Estimation
}

\author{
Julian Kolodko \\ Intelligent Control Systems Laboratory \\ Griffith University \\ Nathan, Q, 4111, AUSTRALIA \\ j.kolodko@sct.gu.edu.au
}

\author{
Ljubo Vlacic \\ Intelligent Control Systems Laboratory \\ Griffith University \\ Nathan, Q, 4111, AUSTRALIA \\ l.vlacic@griffith.edu.au
}

\begin{abstract}
Motion can be a useful sensory dimension for autonomous navigation if it is available in real time. In this paper we present our approach to real time motion processing giving an outline of the algorithm and hardware architecture we have developed. We then describe in more detail how this architecture is being implemented using FPGA technology.
\end{abstract}

\section{Keywords}

Motion Estimation, Data fusion, FPGA.

\section{INTRODUCTION}

If motion is to be a useful sensory dimension for navigating in a dynamic environment, it must be extracted quickly relative to the dynamics of the objects in that environment, i.e. in "real-time". In the context of motion processing "real-time" refers to processing that is quick enough to prevent temporal aliasing [1]. For an autonomous vehicle the result of slow or high latency processing is at best, a poor navigation plan and at worst, a catastrophic collision.

Producing real-time motion estimates is challenging since it typically involves applying some form of iterative search to the massive bandwidth of a live video stream. Furthermore, overheads such as memory management and interfacing must be addressed. In order to overcome these problems we approach the design problem from end to end. That is, care is taken to ensure the chosen algorithm can be implemented efficiently in hardware, and the hardware is chosen to ensure our overall goals can be met.

\section{What Are We Trying To Achieve?}

Our primary goal is to design a compact, self-contained system for computing motion estimates in real time. This system is targeted for use as a part of a navigation system of an autonomous vehicle and is to use a combination of range and visual data in order to reduce ambiguity. The output of the system is a segmented one dimensional motion estimate.

Non Traditional Approaches to Motion Processing When considering motion processing hardware, it is common to assume that the system must digitize a visual image (or range data) and process that image with digital hardware however this is not necessarily the case. For example, the VisionChip [2][3][4] is an approach to vision processing where, rather than using a camera and separate processing hardware, the imaging and processing components are incorporated into a single device. A variety of motion processing algorithms can be used and they are often implemented using analogue components [3] though hybrid digital implementations are also possible [4]. Difficulties still abound in this technology including low resolution and problems integrating video and processing elements into the same silicon.

Other systems are active rather than being strictly passive. For example, structured light can be used to determine motion. In this scenario, a known pattern of light is projected into the environment using a laser. A camera detects the reflected pattern and determines the structure and motion of objects in the environment based on the deformation in that pattern. For example, a laser stripe and camera can be used to determine relative motion between an object and a vehicle [5]. A similar system is used to enable robotic grasping of moving objects [6]. Another active system was proposed by Houghton et al [7]. They show how the speckle pattern that arises when a laser shines on an optically rough surface can be used to measure motion. This idea was developed [8] into and ASIC that is capable of processing 1D speckle images at high speed.

\section{Traditional Approaches to Motion Processing}

There are three broad approaches in traditional motion processing hardware: custom design, video processor based implementations and $\mathrm{PC}$ based implementations.

Motion processing in custom hardware can involve design and implementation at the integrated circuit and circuit board levels. For example, Röwekamp 1997 [9] shows how a high resolution optical flow field can be calculated at very high speed (up to $1500 \mathrm{~Hz}$ for a $128 * 128$ vector field) when a digital ASIC is used. Stoffler 1997 [10] uses MPEG correlation processors to compute a sparse but robust optical flow of up to 525 vectors at 25 frames per second. Lee et al [11] use a mixed signal VLSI neuroprocessor to compute 64 motion vectors and Boluda [12] uses an FPGA and a log polar vision system to identify objects that are moving independently of the camera at $285 \mathrm{~Hz}$.

Video processors are usually purpose built parallel computers or transputers. Examples of implementations using such technology include Mittal et al [13] who shows how time to collision can be computed at $25 \mathrm{~Hz}$ allowing a robot to navigate without collision with static obstacles. In a more complex system, Willersinn 1997 [14] uses a MiniVista transputer and a pair of PowerPC's to compute flow using a local approach based on the second image 
derivatives, cluster the flow to reduce the data rate and perform a final segmentation at $12 \mathrm{fps}$. Szabo 1996 [15] presents a physically large processing system based on the DataCube platform. It has been used for a number of motion estimation tasks including independent motion estimation based on the focus of expansion, gradient based optical flow and correlation based optical flow. The system is capable of running at up to $15 \mathrm{fps}$ depending on the algorithm.

The simplest approach is to use a $\mathrm{PC}$ or a digital signal processing (DSP) system coupled with a frame grabber. Such approaches are becoming more practical as computing power increases. Notable implementations include Benoit 1999 [16] who developed a system that can produce motion estimates at $4 \mathrm{fps}$ on a $100 \mathrm{MHz}$ R4600 SGI workstation using a correlation based approach. Batavia et al 1998 [17] shows how optical flow can be used to monitor a vehicles blind spot at 8-15 frames/second using a Pentium based PC. Camus et al 1996 [18] compute flow divergence for both steering control and collision detection (with static obstacles) of a mobile robot. The entire implementation operates 35 frames per second on a $80 \mathrm{MHz}$ Sparc machine. Krose et al [19] show how a mobile robot can navigate through the center of a corridor using the temporal derivatives of optical flow.

\section{Why aren't these approaches enough?}

It is evident from the previous discussion that modern hardware developments have made real time motion processing more achievable. This begs the question, why are existing approaches not yet sufficient?

The central tenet of our work is that motion segmentation should be performed using a combination of visual and range information in order to minimise ambiguity that arises when visual information alone is used. This ambiguity arises because visual motion is a function of both velocity and depth. None of the implementations listed above directly utilise range data. Algorithms that combine data from both sources exist (eg [20] [21]), however these have not been used in real time implementations and have other drawbacks. Furthermore, none of these implementations are able to segment the visual environment into coherently moving regions, a feature critical for effective navigation planning.

\section{ALGORITHM}

In this section we define what we mean by "real time" in the context of our work and show how a dynamic scale space is used to achieve real time processing. We then introduce our motion estimation algorithm that fuses visual and range data to eliminate motion ambiguity. Our algorithm separates motion estimation from motion segmentation and reduces what is traditionally an expensive 2D minimisation to a less costly $1 \mathrm{D}$ problem.

\section{Real Time and Dynamic Scale-Space}

Gradient based approaches such as ours fail if apparent motion greater than about one pixel per frame is present. Greater motion results in temporal aliasing and this in turn makes the image derivatives that we use to estimate motion invalid. Based on this, we define "real-time" as a rate quick enough to avoid temporal aliasing.

The usual method of resolving the problem of temporal aliasing is to use a scale-space approach where a pyramid of subsampled images is created. The motion estimation algorithm is then applied at each level of the pyramid. High velocities can be reliably measured at higher levels of the pyramid while the lower levels (i.e. those levels with less subsampling) are used to measure lower velocities. Unfortunately this scheme is problematic. Generating and storing an image pyramid is time consuming and propagating motion estimates from one level to the next adds significant complexity [22] making it extremely difficult to implement such a system in real time.

Rather than implementing a full scale-space scheme, we use a dynamic scale-space [1] where an appropriate scale is chosen to avoid temporal aliasing based on range data. The nearest object is likely to have the highest apparent velocity, and it is the object with which we are most likely to collide with in the short term, so we choose a scale that prevents temporal aliasing for that object.

A simple geometric [1] argument based on the pinhole camera model can be used to derive the following relationship between the required frame rate for real time processing, object velocity $(\mathrm{V}=0.1 \mathrm{~m} / \mathrm{s})$ and distance $(\mathrm{D}=0.4 \mathrm{~m})$, and the camera focal length $(f=4.8 \mathrm{~mm})$ and pixel pitch $(\zeta$ $=12.5 \mathrm{~mm}$ )

$$
\text { FrameRate }=\frac{2 V f}{D \zeta} f p s
$$

This is a worst-case relationship based on a maximum relative velocity of $2 \mathrm{~V}$ between the camera and an object. In our environment, with our camera a frame rate of $192 \mathrm{fps}$ is required to achieve real time if dynamic scale space is not used. With a 5 level dynamic scale-space, this falls to a much more attainable $12 \mathrm{fps}$.

\section{Motion Estimation}

Our short range motion estimation algorithm uses the optical flow constraint equation (OFCE [23]), together with the equations of motion [24] to fuse visual and range information [1]. This results in the following constraint equation.

$$
E_{D}=-\frac{f \psi}{Z_{(x, y)}} \mathbf{U}_{\mathbf{x}_{(x, y)}} I_{x_{(x, y)}}+I_{t_{(x, y)}}=0
$$

In this equation, $I_{x}$ and $I_{\mathrm{f}}$ are the horizontal and temporal derivatives of the image sequence, $f$ is the camera focal 
length, $\psi$ is a constant converting $\mathrm{m} / \mathrm{sec}$ to pixel/frame and $Z$ is depth. $\mathbf{U}_{\mathbf{X}}$ is the lateral apparent velocity which corresponds to the speed (in $\mathrm{m} / \mathrm{sec}$ ) at which a point in the image appears to be moving. Since $\mathbf{U}_{\mathbf{X}}$ does not in general correspond to an objects physical velocity its absolute value is irrelevant. This allows us to assume the scale factors $f$ and $\psi$ are equal to one, further simplifying our formulation. The algorithm begins by solving for $\mathbf{U}_{\mathbf{X}}$ at each pixel. To reduce the data stream we take the median estimate for each column. This reduces computational complexity to $\mathbf{O}(i x)$ (where $i$ is the number of iterations, $x$ is the width of the image). Without this reduction computational complexity is $\mathbf{O}(i x y)$ (where $y$ is the height of the image) which is typical of optical flow based methods. The net result is a robust motion estimate for each column which is then segmented using a first order weak string model [22][25] giving us the desired object boundaries.

The weak string results in a minimisation problem that is non-convex. To solve such a problem in using custom digital hardware deterministic algorithms are preferred to stochastic algorithms since they are more easily mapped to digital logic. We use the Graduated Non-Convexity method [22] together with Successive Overrelaxation to find the global minimum.

Implicit in this model is the assumption that the world is piecewise planar with the planes parallel to the cameras sensing surface. The reason is threefold. Firstly, by using image columns as regions of support for the motion estimate we have assumed that each pixel in the column is part of the same object. To minimise the chance of this assumption being violated we use a robust estimate for the motion of each column and we use a relatively narrow image. Secondly, the weak string model allows segmentation of motion effectively allowing piecewise planar world. Finally, because only a single dimensional range scan is available, we assume that range value is constant over the entire image column, hence the planes are parallel to the cameras sensing surface.

\section{OUR HARDWARE IMPLEMENTATION}

In this section a brief introduction to the core components of our system is given before discussion moves to the specific detail of our implementation.

\section{Processing}

In order to accelerate prototype development we have opted for a commercially available prototype development system from Lyr Signal Processing [26]. The key processing element on the SignalMaster platform is an Analog Devices Sharc ADSP-21062 DSP which has access to $16 \mathrm{MB}$ of RAM. A number of interface options are available on the board (e.g. PC104, Ethernet) however the most important for our application is the BITSI interface that allows us to add GatesMaster Mezzanine card. This adds a Virtex XCV800 FPGA running at $40 \mathrm{MHz}$ and an additional $16 \mathrm{MB}$ of SDRAM, allowing greater implementation flexibility. The majority of our system is implemented in FPGA since this allows a compact solution where all processing, glue and interface logic is contained within a single chip.

\section{Fuga 15D Camera}

We use the Fuga15D camera from C-Cam Technologies because its simple RAM-like interface eases development. The Fuga15D has a logarithmic intensity response making it less sensitive to illumination variation, however it has higher noise levels and less contrast than other cameras. Fortunately, the noise pattern is fixed and easily corrected and contrast can be adjusted via gain controls though a trade off must be found between sufficient contrast and sensitivity to illumination variation.

A further issue with this camera is that is does not use a shutter. The Fuga camera measures the value of a pixel only when that pixel is addressed while traditional cameras effectively measure the value of all pixels at the same time - that is, when the shutter closes. Thus, if there is a significant delay addressing pixels, the resulting image may contain motion distortion appearing as shear in the shape of moving objects. We have found that in our context, motion distortion is negligible.

\section{ICSL Vehicle Testbeds}

Our system is being tested using the ICSL robots [27]. The ICSL has developed a number of vehicle test-beds for use in evaluating autonomous vehicle concepts without the need for large scale testing facilities. These robots feature a distributed multi-microcontroller architecture where the PIC $16 \mathrm{C} 74$ microcontroller and the $\mathrm{I}^{2} \mathrm{C}$ serial bus are the primary building elements. Subsystems include infrared and ultrasonic ranging systems for navigation, radio packet modem for communication and a laser based system for intelligent speed adaptation. A number of behaviors have been developed for these test-beds including fuzzy logic based leader following, static obstacle avoidance, lane keeping, intersection navigation and overtaking. Maximum velocity for the robots is $0.1 \mathrm{~m} / \mathrm{s}$.

\section{IMPLEMENTATION DETAILS}

Figure 1 illustrates the architecture of our implementation. To allow for testing, a link to a PC allows for visualization of results though this link can easily be replaced by another device if further processing is required. This design consists of three key sections; the memory subsystem, a collection of processes (i.e. reading from camera, processing data, output to PC etc) and buffers designed to mediate communication between processes and memory.

\section{RAM Interface and Memory Management}

Because this system a number of processes each of which could potentially require simultaneous access to RAM, bus arbitration is a necessity. Further, careful memory management (RAM allocation) leads to more efficient RAM use since devices use RAM differently. 


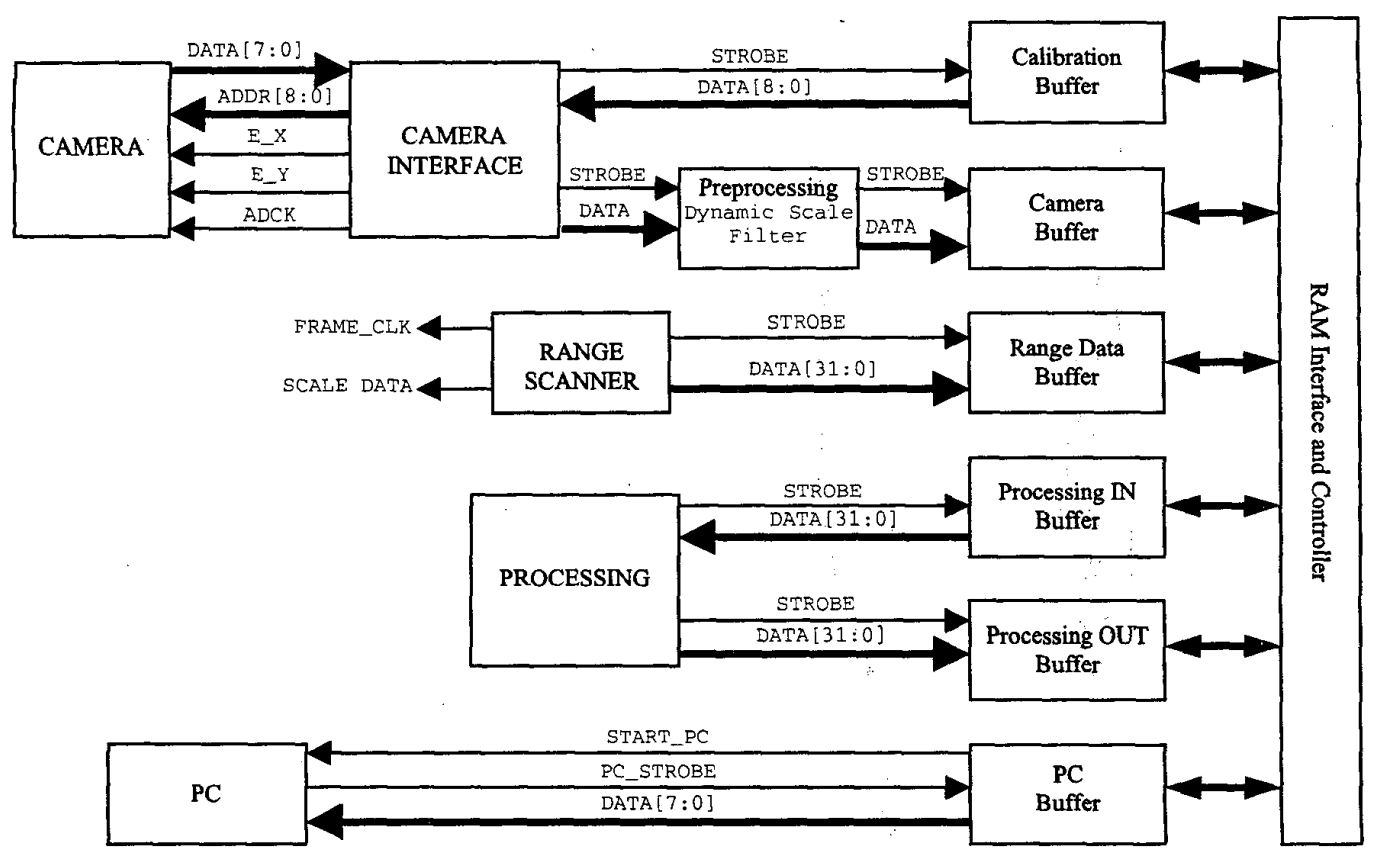

Figure 1. System Architecture

Bus arbitration is implemented via a combination of decoupling buffers and the RAM Interface and Controller (RAMIC) module. Each process has a decoupling buffer implemented using Block SelectRAM (this RAM is within the FPGA so buffering does not cause further contention). The buffers allow each process to operate at full speed without waiting on memory. This is especially critical for the FUGA camera where we must maintain a constant pixel rate to avoid distortion. The RAMIC polls each buffer in a round robin fashion allowing each buffer appropriate access to RAM and placing an absolute upper bound on RAM access times. To maintain a constant pixel rate, buffers do not pause when an overflow/underrun occurs. In this situation it is important to know the upperbound on memory access time so that the design can be made overflow/underun free.

Aside from providing a low level interface to SDRAM, address decoding and bus arbitration, the RAMIC also provides a number of composite operations that allow more efficient RAM use. For example, we exploit the fact that data from the Fuga camera is 8 bits wide, while each SDRAM location is 32 bits wide to optimize memory access. A section of RAM is allocated as a "frame-buffer" with a one to one mapping from camera to RAM addresses, however we stack pixels so that each RAM location stores four frames of data. To implement this, existing pixel data is read from RAM, shifted 8 bits to the left and the new pixel is inserted in the least significant eight bits. From the point of view of the buffer, this is a single "write pixel" operation, which is more efficient than using the polling process to perform the implied read and write separately.
However the real efficiency is realized when data is read for processing. Our algorithm requires 3 frames of data at each location to compute temporal image derivatives. With our pixel stacking scheme, this data can be obtained with a single RAM read operation.

The final function of the RAMIC is to generate zero-order calibration data for the FUGA camera. When the system is first started, the RAMIC generates a single image by taking the pixel-wise average of 16 frames of data. During this time a plain, translucent sheet of paper covers the lens so that the image seen by the camera contains only noise. Next, the distribution of pixel intensities is shifted so that is has zero mean. The resulting image represents the noise at each pixel called the calibration value. The corresponding calibration value is subtracted from each pixel to eliminate the fixed noise pattern.

\section{Buffers}

The Virtex FPGA has dedicated RAM (known as Block SelectRAM) available on board. SelectRAM provides 4096 bits of storage, is dual ported, and the data width of each port can be chosen independently. A total of 28 blocks of SelectRAM are available on our XVC800 Virtex device. Because we use SelectRAM for decoupling buffers we are able to implement buffers that can simultaneously be accessed by a process and by the RAMIC.

The specific design of buffers varies depending on their task though they all have the general structure shown in figure 2. The RAMSide controller mediates operations between SelectRAM and the RAMIC. Fata and address lines between Buffers and the RAMIC are under tristate control. The handshake between a buffer and the RAMIC 


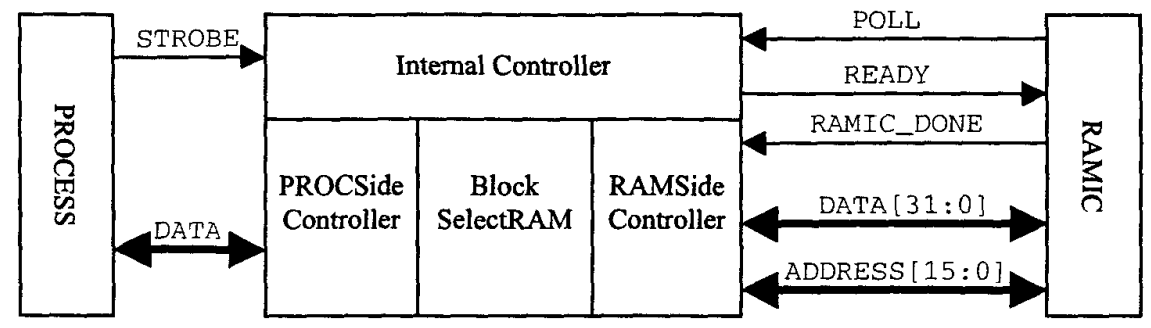

Figure 2. Structure of Buffers

begins when the RAMIC sets a buffers polls line high. If that buffer has an operation pending, the RAMSide controller will assert the READY line and take control of the busses. The RAMIC now begins the requested operation and will assert the RAMIC_READY line when the operation is complete. Note that only a single operation is performed during a single poll, though this can be a compound operation (such a stacking a pixel into RAM).

The PROCSide controller provides access to the buffer to a single process. Because the time to access the buffer is known these controllers do not use a two phase handshake like the RAMSide controller. Instead, the processes simply STROBEs the controller when an operation is required and the operation is completed one clock cycle later.

The internal controller is a logical device whose implementation is spread across both the RAMSide and PROCSide buffers. The primary purpose of this device is to maintain buffer pointers and generate RAM addresses. In some buffers, this controller also performs some simple processing on the data stream.

\section{Camera Datapath}

In the camera data-path the Camera Interface generates the control signals necessary to read a $512 * 32$ pixel image from the camera. E_X and E_Y are the $X$ and $Y$ address strobes respectively, $A D D R$ contains the address and $A D C K$ (active low) is the analogue to digital conversion strobe and is controlled by a $1 \mathrm{MHz}$ pixel clock. Frame timing is controlled by the $10 \mathrm{~Hz}$ FRAME_CLK signal from the Range Scanner Interface. The camera interface also applies zeroorder calibration to the raw camera data. The preprocessing block then takes the calibrated image, subsamples it appropriately for the current dynamic scale space. This subsampled image is fed into a queue (as per [9]) where a low pass filter is applied and the result is passed to the camera buffer. Data is written into RAM sequentially from address 0 up to address $(512 * 32)-1$ in row major order.

\section{Range Scanner}

The range scanner block implements the interface to a range scanning device and provides support operations such as mapping the range data onto the camera coordinate system, and provision a frame clock and of scale data. Our target range scanner operates at $10 \mathrm{~Hz}$ and this rate will be used as the frame clock. This is slightly slower than our real-time rate of $12 \mathrm{~Hz}$ however $10 \mathrm{~Hz}$ is acceptable since, in practice, we will rarely experience the worst case conditions assumed in our temporal aliasing calculations. Scale data relates to the image width at the current scale and other related parameters.

\section{Processing}

Our system is designed so that the reading of new image and range data occurs concurrently with the output of data to PC. When the buffering of this data is complete and when scale data has been updated, processing begins. The ProcessingIN buffer computes image derivatives and these are used by the processing block to generating a robust estimate of motion for each image column. This one dimensional motion estimate is then smoothed and segmented using a weak string model to produce the final motion estimate and segmentation.

\section{Output Process}

Because both the output of existing data to the PC and input of new from the camera and range finder occur concurrently, care must be taken to ensure data is not clobbered. We achieve this by ensuring that output to the PC always leads input of new data so that data is only updated once it has been output. To prevent possible buffer underruns, the PC Buffer reads ahead slightly before triggering the PC to start accepting data (via the START_PC line). The PC (or other downstream device such as a DSP) is able to read a new value from the buffer by toggling the PC_STROBE line.

\section{Timing and Resource Use}

Our current implementation is complete except for the processing block and range scanner interface. This implementation has utilized approximately $10 \%$ of available logic resources, 35 digital IO's and 8 blocks of SelectRAM. Our implementation allows IO operations for the camera, $\mathrm{PC}$ and the laser range finder to be interleaved, however data processing must occur independently. Since $1 / O$ operations require $22.5 \mathrm{~ms}$ per frame, we have $77.5 \mathrm{~ms}$ available for processing giving a clock cycle budget of 19 clocks per pixel per iteration under the assumption of $512 * 32$ pixel images and 10 iterations per frame. This is more than sufficient for our algorithm.

\section{ACKNOWLEDGMENTS}

We would like to thank Frauenhofer Autonomous Intelligent Systems Group (AiS) for their generous donation of 
the Signal Master platform and Fuga Camera used in this work.

\section{CONCLUSIONS}

In this presentation we have introduced a motion estimation algorithm tailored for real time implementation then shown how this algorithm can be implemented in a single chip together with all glue and interface logic to produce a compact sensing solution.

\section{REFERENCES}

[1] Kolodko, L. Vlacic, "From Motion Processing to Autonomous Navigation", To appear in Intelligent Robots: Vision, Learning and Interaction, KIAST Press, 2003

[2] Moini A., "Vision Chips or Seeing Silicon", Centre for High Performance Integrated Technologies and Systems, Department of Electrical \& Electronics Engineering, The Univ. of Adelaide, Australia. http://www.eleceng.adelaide.edu.au/Groups/GAAS/Bugeye/ visionchips/vision chips/vision chips.html

[3] Luthon F., Popescu G. V., Caplier A., "An MRF Based Motion Detection Algorithm Implemented on Analog Resistive Network', ECCV 94, LNCS vol 800, Jan-Olof Eklundh (ed), pp 167-174, 1994

[4] Dron L. G., "Computing 3D Motion in Custom Analog and Digital VLSr", Ph.D. Thesis, MIT AI Lab, AI Tech Rep 1498, August 1994

[5] Monteiro D., Jouvencei B., “Visual Servoing for Fast Mobile Robot: Adaptive Estimation of Kinematic Parameters", IECON '93, vol 3, pp 1588-1593, 1993

[6] Zhu L., Fujimoto H., Sano A., Yamakawa S., "Adaptive Visual Tracking of Moving Objects with Neural PID Controller", Proc SPIE, vol 4197, pp 340-350, 2000

[7] Houghton A. D., Mawer J. R., Ivey P. A., “ASIC for High Resolution Motion Sensing", Electronics Letters, Vol 31(8), pp 635-636, April 1995.

[8] Houghton A., Rees G., Ivey P., "A method for processing laser speckle images to extract high-resolution motion", Measurement Science and Technology, Vol 8(6), pp 611617, Jun 1997

[9] Röwekamp T., "A Smart Sensor System for Real-Time Optical Flow Estimation", Ph.D. Thesis, Technischen Universität Cottbus, 1997.

[10] Stoffler N. O., Schnepf Z., An MPEG-Processor-Based Robot Vision System for Real-Time Detection of Moving Objects by a Moving Observer, http://www.lpr.ei.tum.de/ stoffler, Cited Jan 2000
[11] Lee J-C., Sheu B. J., Fang W-C., Chellappa R., "VLSI Neuroprocessor for Video Motion Detection", IEEE. Trans. On Neural Networks, vol 4(2), pp 178-191, March 1993.

[12] Boluda J. A., Blasco F. J., Pardo F., “A Scalable Reconfigurable FPGA-Based Architecture for Robotic Navigation"

[13] Mittal A., Valilaya A., Banerjee S., Balakrishnan M., "Real Time Vision System for Collision Detection"

[14] Willersinn D., Enkelmann W., "Robust Obstacle Detection and Tracking by Motion Analysis", IEEE Conference on Intelligent Transportation System, pp 717-722, 1997.

[15] Szabo, S., Coombs, D., Herman, M., Camus, T., Liu, H., " $A$ Real-Time Computer Vision Platform for Mobile Robot Applications", The Joumal of Real-Time Imaging, Academic Press, Vol. 2, No. 5, pp. 315-327, October 1996

[16] Benoit S. M., "Monocular Optical Flow For Real-Time Vi. sion Systems", Ms Thesis, Dept. Elect. Eng., Center for Intelligent Machines, McGill University, Canada, 1999.

[17] Batavia P. H., et al, "Overtaking Vehicle Detection Using. Implicit Optical Flow", IEEE Conference on Intelligent Transport Systems, pp 729-734, 1997

[18] Camus T., Coombs D., Herman M., Hong T-H., "Real-Time Single-Workstation Obstacle Avoidance using only WideField Flow Divergence", $13^{\text {th }}$ Int. Conf. Pattern Recognition - Applications and Robotic Systems, August 1996.

[19] Krose B., Dev A., Benavent X., Groen F., "Vehicle Navigation on optic flow"

[20] Nygards J., Wenersson A., "Model Based Fusion of Laser and Camera: Range Discontinuities and Motion Consistency", Proc. $3^{\text {rd }}$ Int. Conf. Information Fusion, vol 1, pp TUB5/16-21 2000

[21] Spies H., Jahne B., Barron J. L., "Dense Range Flow from Depth and Intensity Data", International Conference on Pattern Recognition, pp 131-134, 2000.

[22] Black M. J., "Rabust Incremental Optical Flow", Ph.D. Thesis, Yale, 1992.

[23] Horn and Schunk, "Determining Optical Flow", MT Artificial Intelligence Lab, AI Memo 572, April 1980

[24] Mitiche A., "Computational Analysis of Visual Motion", New York, Plenum Press, ISBN: 0-306-44786-X, 1994

[25] A. Blake, A. Zisserman, "Visual Reconstruction", MIT Press, 1987, ISBN 0-262-02271-0

[26] http://www.signal-1sp.com/

[27] Vlacic Lj., Engwirda A., Kajitani M., "Cooperative behaviour of Intelligent Agents; Theory and Practice", Soft Computing and Intelligent Systems, ed. N. K. Sinha, M. M. Gupta, Academic Press, pp 279-307, 2000 